\title{
SOME REMARKS ON THE SEQUENCES OF EXHAUSTIVE MEASURES AND UNIFORM BOUNDEDNESS OF MEASURES
}

\author{
SURJIT SINGH KHURANA
}

\begin{abstract}
Nikodym-type theorems and uniform boundedness theorems for measures on Boolean algebra with interpolation property are derived from corresponding results for $\sigma$-algebras.
\end{abstract}

In [3], [6], some Vitali-Hahn-Saks and Nikodym-type theorems are proved about the measures on a Boolean algebra $\mathfrak{U}$ with the interpolation property, called the property I. In this note we prove that these results are simple consequences of well-known results.

For a Hausdorff locally convex space $E$, always assumed to be over $K$, the field of real or complex numbers, $E^{\prime}$ will denote its topological dual [4]. A Hausdorff Abelian topological group $G$ is called normed if there exists a function $|\cdot|: G \rightarrow[0, \infty)$ satisfying the conditions (i) $|x|=0 \Leftrightarrow x=0$, (ii) $|x+y|<|x|+|y|$, such that the topology of $G$ comes from the metric $P$, $P(x, y)=|x-y|[1]$. A Boolean algebra $\mathfrak{A}$ is said to have the property $\mathrm{I}$ if for any two sequences $\left\{x_{n}\right\},\left\{y_{m}\right\}$ in $\mathfrak{A}$ with $x_{n} \uparrow, y_{m} \downarrow$, and $x_{n}<y_{m}, \forall m, n$, there exists an $x \in \mathfrak{U}$ such that $x_{n} \leqslant x \leqslant y_{m}, \forall m, n$ [5]. We shall use the theory of submeasures developed in [1] (in [1], submeasures are defined on the algebra of subsets of a set; by considering the clopen subsets of the Boolean space of a Boolean algebra, they can be defined on a Boolean algebra).

LemMA 1. Let $\mathfrak{A}$ be a Boolean algebra with the property I, $\mu: \mathfrak{A} \rightarrow[0, \infty) a$ finite submeasure and $\eta=\{x \in \mathfrak{U}, \mu(x)=0\}$. Then $\mathfrak{U} / \eta$ is a $\sigma$-complete Boolean algebra.

Proof is very straightforward and similar to [5, Lemma 3.3, p. 275], and as such is omitted.

LEMMA 2. Let $2^{N}$ be the set of all subsets of $N$, the set of natural numbers, and $\nu_{n}: 2^{N} \rightarrow G$ be a sequence of exhaustive finitely additive set functions such that $\lim \nu_{n}(M)$ exists, $\forall M \in 2^{N}$. G being a Hausdorff Abelian topological group. Then $\left\{\nu_{n}\right\}$ are uniformly exhaustive.

Received by the editors January 27, 1978.

AMS (MOS) subject classifications (1970). Primary 46G 10; Secondary 28A45, 28A60.

(C) 1979 American Mathematical Society 0002-9939/79/0000-0063/\$01.50 
This is proved in [2, Theorem (BJ), p. 726].

TheOREM 3 [3, Theorem 3.1, p. 111]. Let $\mathfrak{A}$ be a Boolean algebra with the property I, $G$ a Hausdorff Abelian topological group, and $\mu_{n}: \mathfrak{X} \rightarrow G$ a sequence of exhaustive (三 strongly bounded, [1]) finitely additive set functions such that $\lim \mu_{n}(b)$ exists for every $b \in \mathfrak{A}$. Then $\left\{\mu_{n}\right\}$ are uniformly exhaustive.

PROof. Since every group is a subgroup of a product of normed groups, we can assume that $(G,|\cdot|)$ is normed. Every $\mu_{n}$ gives a submeasure $\dot{\mu}_{n}$,

$$
\dot{\mu}_{n}(b)=\sup \left\{\left|\mu_{n}(a)\right|: a \in \mathfrak{A}, a \leqslant b\right\}
$$

which is exhaustive and finite [1, II, Corollary 4.11, p. 279]. The submeasure

$$
\mu=\sum_{n=1}^{\infty} \frac{1}{2^{n}\left(1+\dot{\mu}_{n}(1)\right)} \dot{\mu}_{n}
$$

is finite and exhaustive. By Lemma $1, \mathfrak{A}_{0}=\mathfrak{A} / \eta$ is a $\sigma$-complete Boolean algebra with $\eta=\{x \in \mathfrak{U}: \mu(x)=0\}$. Let $x \rightarrow \hat{x}$ be the canonical mapping $\mathfrak{U} \rightarrow \mathfrak{U}_{0} . \quad \bar{\mu}_{n}: \mathfrak{U}_{0} \rightarrow G, \bar{\mu}_{n}(\hat{x})=\mu_{n}(x)$ is well defined. Fix a disjoint sequence $\left\{y_{i}\right\}$ in $\mathfrak{A}$ and define $\nu_{n}: 2^{N} \rightarrow G, \nu_{n}(M)=\bar{\mu}_{n}\left(\bigvee_{i \in M} \hat{y}_{i}\right)$. Since $\nu_{n}$ are exhaustive (this follows from the fact that for any disjoint sequence $\left\{M_{j}\right\}$ in $2^{N}$, $\left\{\bigvee_{i \in M_{j}} \hat{y}_{i}\right\}$ are disjoint), and $\lim \nu_{n}(M)$ exists, $\forall M \in 2^{N}$, they are uniformly exhaustive, by Lemma 2 . The result now follows.

TheOREM 4 [3, Theorem 2.1, p. 105]. Let $\mathfrak{A}$ be a Boolean algebra with the property I, $E$ a Hausdorff locally convex space, and $H$ a set of bounded finitely additive set functions $\mathfrak{A} \rightarrow E$ such that $\{\lambda(b): \lambda \in H\}$ is a bounded subset of $E$, for every $b \in \mathfrak{A}$. Then $P=\{\lambda(b): \lambda \in H, b \in \mathfrak{A}\}$ is bounded in $E$.

Proof. Fix $f \in E^{\prime} . f \circ H$ is a set of bounded finitely additive, scalar-valued set functions on $\mathfrak{A}$ and are pointwise bounded on $\mathfrak{A}$. By [5, Theorem 3.2] $\{f \circ \lambda(b): \lambda \in H, b \in \mathfrak{A}\}$ is bounded. This means $P$ is weakly bounded in $E$ and so is bounded in $E$ [4].

\section{REFERENCES}

1. L. Drewnowski, Topological rings of sets, continuous set functions, integration. I, II, Bull. Acad. Polon. Sci. Sér. Sci. Math. Astronom. Phys. 20 (1972), 269-276, 277-286.

2. __ Equivalence of Brooks-Jewett, Vitali-Hahn-Saks and Nikodym theorems, Bull. Acad. Polon. Sci. Sér. Sci. Math. Astronom. Phys. 20 (1972), 725-731.

3. B. T. Faires, On Vitali-Hahn-Saks-Nikodym theorems, Ann. Inst. Fourier (Grenoble) 26 (1976), 99-114.

4. H. H. Schaefer, Topological vector spaces, Macmillan, New York, 1971.

5. G. Seever, Measures on F-spaces, Trans. Amer. Math. Soc. 133 (1968), 267-280.

6. B. T. Faires, On Vitali-Hahn-Saks type theorems, Bull. Amer. Math. Soc. 80 (1974), 670-674.

Department of Mathematics, University of Iowa, Iowa City, Iowa 52242 